\title{
Fabrication and Characterization of Organoclay Reinforced Polyester Based Hybrid Nanocomposite Materials
}

\author{
Noor Zaman \\ Institute of Chemistry \\ Shah Abdul Latif University \\ Khairpur, Pakistan \\ noorzamanshar@gmail.com \\ Attique Ur Rehman \\ Institute of Geology \\ University of The Punjab \\ Lahore, Pakistan \\ attique786aur@gmail.com
}

\author{
Shfique Ahmed \\ Institute of Chemistry \\ Shah Abdul Latif University \\ Khairpur, Pakistan \\ ashafique2010@gmail.com \\ Abdul Raheem Shar \\ Institute of Chemistry \\ Shah Abdul Latif University \\ Khairpur, Pakistan \\ saifiraheem3863920@gmail.com
}

\author{
Muhammad Sanaullah \\ Institute of Geology \\ University of the Punjab \\ Lahore, Pakistan \\ sana.ullah.geo@pu.edu.pk
}

\author{
Muhammad Ramzan Luhur \\ Dpt of Mechanical Engineering \\ QUEST Nawabshah \\ Pakistan \\ ramzanluhur@yahoo.com
}

\begin{abstract}
Synthesis and characterization of polyester nanocomposites was conducted in order to fabricate hybrid composite materials of polyester/montmorillonite (MMT). Polyester based polymeric nanocomposite materials were synthesized by incorporating MMT nanoclay to produce polyester/MMT hybrid materials. Successful efforts were made to fabricate hybrid nanocomposite materials based on matrix (polyester based) and reinforcement (organoclay) through sonication at 6 and 12 hours. Synthesized nanocomposite polymers (polyester/MMT) showed different properties when compared to the properties of MMT and polyester, which confirmed the successful fabrication of the desired material. The finest incorporation of polyester with MMT was verified by UVVisible spectrophotometer, Fourier tranform-infrared (FTIR) and scanning electron microscopy (SEM). The disappearance of the $\mathrm{Si}-\mathrm{O}$ characteristic peak was observed in the FTIR spectrum justifying the fabrication of the desired composite materials. Colored SEM images were used to confirm the fine homogenous distribution of organoclay. Black SEM images showed the matrix and reinforcement together. SEM, FTIR and UV-Visible spectroscopic techniques were used to analyze polyester based nanocomposite materials and organoclay was found randomly distributed in the polymeric matrix whereas on the surface was observed to be mostly uniform.
\end{abstract}

Keywords-polyester; nanocomposite; reinforcement; $U V$-Visible spectrophotometer

\section{INTRODUCTION}

Nano devices are usually used to describe the structures in which nanomaterials are present $[1,2]$. Various subfields are included in nanomaterial field which in which studied substances possess exceptional properties arising from their nanoscale sizes [3]. Nanoionics and nanoelectronics are associated to nanomaterials with speedy transport of ions. Current applications of nanomaterials consist of biosensors, drug delivery and tissue engineering [4].Various technologies are now able to produce nanomaterials smaller than $100 \mathrm{~nm}$ which fell down from predictable solid state silicon methods for manufacturing microprocessors $[5,6]$. It is due to nanotechnology that various products have become cheaper, faster and easier [7]. Manufacturing and utilizing of nanomaterials on industrial scale may have multiple effects on human health and the environment as recommended by nanotoxicology investigations $[8,9]$. Composite materials can be produced by adding of two or more component substances which possess different physical and chemical properties, but on combination may give a substance possessing properties combining the ones of their individual constituents. Within the ending product constituents remain isolate and discrete. Rigidity is generally added by reinforcement which obstructs crack transmission to large extent. Extremely high strength is provided by thin fibers. Therefore overall properties of composites are progressed by mechanical mixing to the matrix. Present study regards UV-Visible and FTIR spectra of the synthesized hybrid nanocomposite matrix. The synthesized composite will be subjected to SEM in order to determine its surface morphology, distribution, compatibility and reinforcement.

\section{METHODS}

Experimental work was conducted to produce hybrid composite materials of polyester synthesis and their characterization. Sonicator and centrifuge machine was used for the synthesis of polyester nanocomposite hybrid materials whereas UV-Visible spectrophotometer, FTIR and SEM instruments were used for their characterization which revealed and verified the optimum incorporation of polyester with MMT. 


\section{A. Synthesis of Polyester/MMT Clay Composite}

For the synthesis of Polyester/MMT clay composite samples the solution was sonicated for 3 to 12 hours with time interval of 3 hours until splendid experimental results. For successful incorporation, clear nanofabrication was found at 9 hours and 12 hours, whereas sample collection was done at 3, $6,9,12$ hours. Before ultra-sonication the color of the sample was white which was then altered to grey jellylike which verified the successful incorporation of organoclay in the polyester.

\section{B. Sonication}

It is also known as ultrasonication as ultrasonic frequencies of greater than $20 \mathrm{kHz}$ are commonly employed [10]. Sonochemistry explains the chemical influences of ultrasound waves on chemical structure $[11,12,13]$.

\section{Centrifuge}

Centrifuge machines used in the laboratories are employed for the purpose of separating heavy particles which settle down at the bottom whereas lighter particles float at the top [14]. By very high speed centrifuge, fine particles may be separated out to nano-scale level as well as molecules having different molecular weights.

\section{Scanning Electron Microscope}

Better than $1 \mathrm{~nm}$ resolution is produced by SEM [15]. The general SEM mode is the determination of subsidiary electrons released by atoms excited by electron beams. An image showing the topography of the sample surface is created by using a special detector, by scanning the sample as well as accumulating secondary released electrons.

\section{RESULTS AND DISCUSSION}

\section{A. UV-Visible Spectroscopic Measurements}

The hybrid composite solution was scanned from $250 \mathrm{~nm}$ to $400 \mathrm{~nm}$ and the $\lambda \max$ at was found to be $341 \mathrm{~nm}$. At this $\lambda \max$, the absorbance of solutions of different concentrations was determined using UV-Visible spectrophotometer.

\section{B. FTIR Characterization}

The spectrum revealed the characteristic peak of organoclay wave numbers at $994.4 \mathrm{~cm}^{-1}$ and $3300-3200 \mathrm{~cm}^{-1}$ which are attributed to the peaks of Si-O stretch vibrations and primary amine $-\mathrm{NH}_{2}$. The value of $994.4 \mathrm{~cm}^{-1}$ is the major characteristic peak for the MMT organoclay. When the MMT is incorporated in polymer matrix through sonication at different time intervals the peak at $994 \mathrm{~cm}^{-1}$ should appear as the confirmation of organoclay presence in the polymer matrix. Above $3400 \mathrm{~cm}^{-1}$, peak broadening justifies the presence of $\mathrm{H}$-bonding. However peaks at $2850.8-2925 \mathrm{~cm}^{-1}$ attribute to the $-\mathrm{CH} 3$ asymmetric stretching (Figure 2).

\section{Comparison of FTIR Spectra of Organoclay at Different Ultrasonication Time}

FTIR spectra of organoclay at different times of sonication confirm the incorporation of MMT in the polymeric matrix through the presence of the Si-O characteristic peak. Interestingly, by increasing sonication time the $\mathrm{Si}-\mathrm{O}$ intensity decreases, further confirming the dispersion of clay into the matrix. This trend is confirmed justified in SEM. The SEM studies clearly indicate the dispersion of clay homogenously with the increase of sonication time. The decrease of Si-O peak and increase of $-\mathrm{OH}, \mathrm{NH}_{2}$ absorption peaks from 3400 to $3600 \mathrm{~cm}^{-1}$ proves the fabrication of the desired composite material.

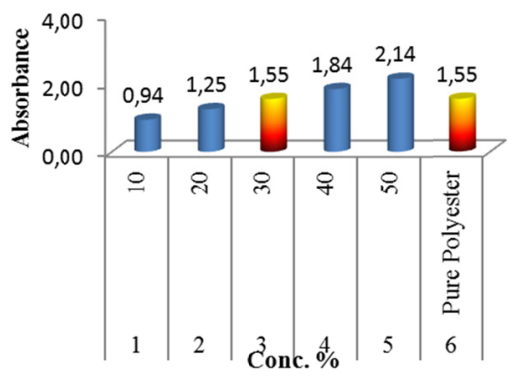

Fig. 1. Concentration of 10, 20, 30, 40 and 50\% of hybrid composite materials

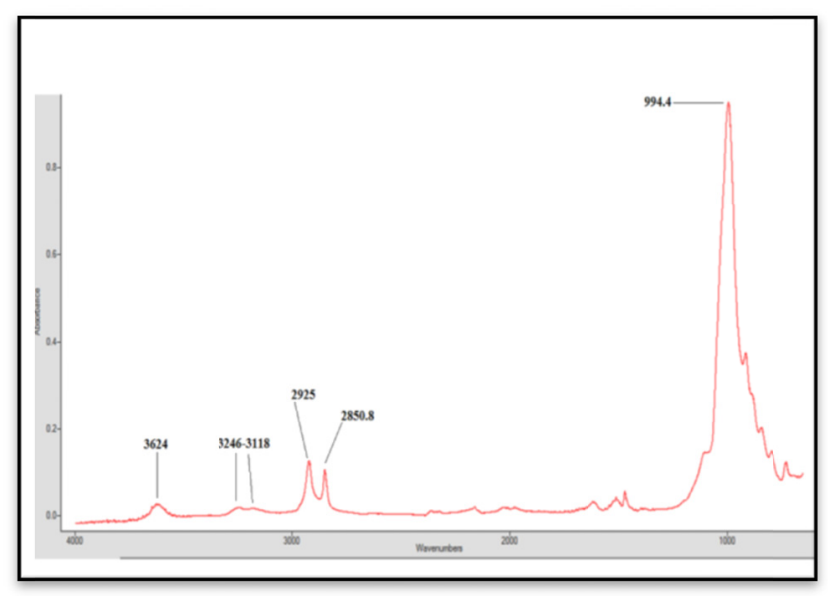

Fig. 2. FTIR spectrum of MMT organoclay

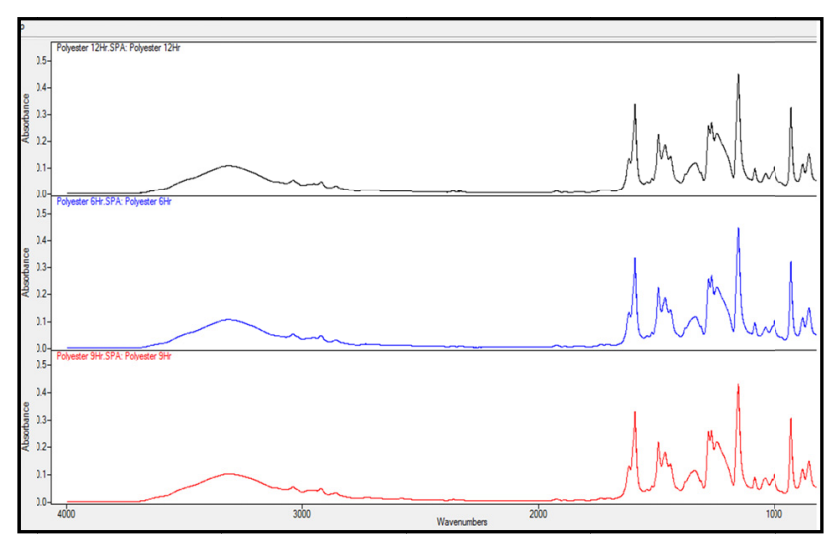

Fig. 3. Comparison of FTIR spectrum of MMT (organoclay) incorporated polyester at $6,9 \& 12$ hours of ultrasonication. 


\section{SEM Characterization}

SEM images of MMTs clearly indicate that it is homogeneous and its surface is smooth (Figure 4). The white spots on the image show the presence of MMT, however the distribution is clustered and not uniform. Black SEM image shows the febricula distribution of organoclay over the polyester. The orange and cool blue SEM images reveal the fine distribution of organoclay. SEM images also show that the surface of MMT and polyester is homogeneous and smooth. Therefore these samples are regarded as pure and fine. Successful incorporation of organoclay MMT was observed which can be seen from SEM images taken at different resolutions. The continuous trend of homogeneity, uniformity and exfoliation is observed at 12 hour of sonication. At 12 hours of sonication the incorporation of MMT organoclay was observed perfectly homogeneous and uniform (Figure4). FTIR spectra and SEM images clearly indicate the incorporation of MMT organoclay regarding our purpose to fabricate hybrid nanocomposite materials based on matrix (polyester based) and inforcement (organic clay) through sonication.
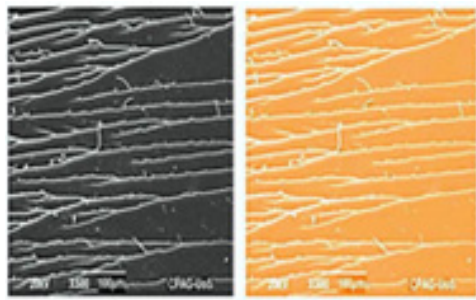

(a)
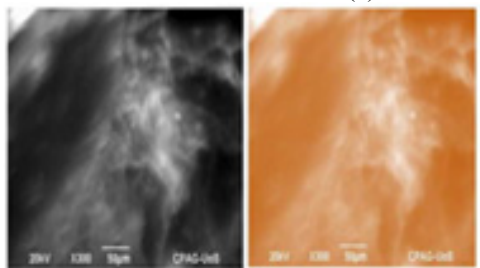

(b)
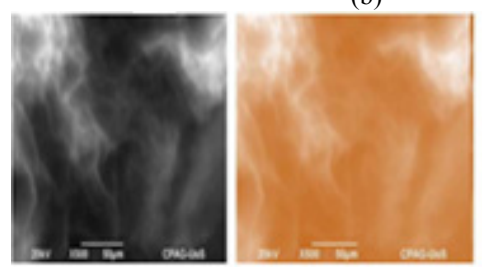

(c)
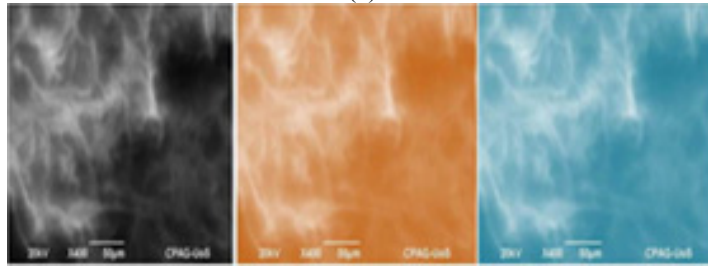

(d)

Fig. 4. (a) SEM images taken at different resolutions showing fine and pure MMTs. SEM images of MMT incorporated polyester at (b) 6 hours, (c) 9 hours and (d) 12 hours of ultra-sonication

\section{CONCLUSIONS}

Regarding our aims and objectives, successful efforts were made to fabricate hybrid nanocomposite materials based on matrix (polyester based) and reinforcement (organic clay) through sonication. The desired nanocomposite hybrid materials were characterized by UV-visible, infra-red and SEM spectroscopy. The FTIR spectra, confirmed the incorporation of organoclay in the polymeric structure of polyester (the disappearance of $\mathrm{Si}-\mathrm{O}$ characteristic peak justifies the fabrication of desired composite materials). The colored SEM images were used to verify the fine homogenous distribution of organoclay whereas black SEM images showed the matrix and reinforcement together. The UV-V polymer spectra are different from the ones of the hybrid nanocomposite materials and on organoclay reinforcement the absorbance got decreased and then increased on increasing concentration of the desired composite material. The column and radar graph show the absorbance variations against concentration.

\section{REFERENCES}

[1] F. Allhof, P. Lin, D. Moore, What is nanotechnology and why does it matter, Wiley-Blackwell, 2010

[2] S. K. Prasad, Modern Concepts in Nanotechnology, Discovery Publishing House, 2008

[3] R. J. Narayan, P. N. Kumta, C. Sfeir, D. H. Lee, D. Choi, D. Olton, "Nanostructured Ceramics in Medical Devices Applications and Prospects", JOM, Vol. 56, No. 10, pp. 38-43, 2004

[4] H. Cho, E. Pinkhassik, V. David, J. M. Stuart, K. A. Hasty, "Detection of early cartilage damage using targeted nanosomes in a post-traumatic osteoarthritis mouse model", Nanomedicine, Vol. 11, No. 4, pp. 939946, 2015

[5] P. Kerativitayanan, J. K. Carrow, A. K. Gaharwar, "Nanomaterials for Engineering Stem Cell Responses", Advanced Healthcare Materials, Vol. 4, No. 11, pp. 1600-1627, 2015

[6] R. C. Shetty, "Potential pitfalls of nanotechnology in its applications to medicine: immune incompatibility of nanodevices", Med Hypotheses, Vol. 65, No. 5, pp. 998-9999, 2005

[7] M. H. Kafshgari, N. H. Voelcker, F. J. Harding, "Applications of zerovalent silicon nanostructures in biomedicine", Nanomedicine (Lond), Vol. 10, No. 16 pp. 2553-2271, 2015

[8] A. Cavalcanti, B. Shirinzadeh, R. A. Freitas, L. C. Kretly, "Medical Nanorobot Architecture Based on Nanobioelectronics", Recent Patents on Nanotechnology, Vol. 1, pp. 1-10, 2007

[9] M. Boukallel, M. Gauthier, M. Dauge, E. Piat, J. Abadie, "Smart microrobots for mechanical cell characterization and cell convoying", IEEE Transactions on Biomedical Engineering,Vol. 54, No. 8, pp. 15361540, 2007

[10] K. S. Suslick, "Sonochemistry”, Science, Vol. 247, pp. 1439-1445, 1990

[11] A. S. Peshkovsky, S. L. Peshkovsky, S. Bystryak, "Scalable high-power ultrasonic technology for the production of translucent nanoemulsions", Chemical Engineering and Processing Process Intensification, Vol. 69, pp. 77-82, 2013

[12] A. Golmohamadi, "Effect of ultrasound frequency on antioxidant activity, total phenolic and anthocyanin content of red raspberry puree", Ultrasonics Sonochemistry, Vol.20, No. 5, pp. 1316-23, 2013

[13] N. S. Deora, N. N. Misra, A. Deswal, H. N. Mishra, P. J. Cullen, B. K. Tiwari, "Ultrasound for improved crystallization in food processing", Food Engineering Reviews, Vol. 5, No. 1, pp. 36-44, 2013

[14] S. R. Mikkelsen, E. Cortón, Bioanalytical Chemistry, Ch 13.Centrifugation Methods, John Wiley \& Sons, pp. 247-267, 2004

[15] J. D. Stokes, Principles and Practice of Variable Pressure Environmental Scanning Electron Microscopy (VP-ESEM), John Wiley \& Sons, 2008 